\title{
DEVELOPMENT AND EVALUATION OF A UAV BASED MAPPING SYSTEM FOR REMOTE SENSING AND SURVEYING APPLICATIONS
}

\author{
C. Eling, M. Wieland, C. Hess, L. Klingbeil, H. Kuhlmann
}

Institute of Geodesy and Geoinformation, University of Bonn, 53115 Bonn, Germany -

(eling, wieland, klingbeil)@igg.uni-bonn.de, s7chhess@uni-bonn.de, heiner.kuhlmann@uni-bonn.de

KEY WORDS: Direct georeferencing, UAV, RTK GPS, GPS Compass, IMU, Magnetometer, Camera synchronization

\begin{abstract}
:
In recent years, unmanned aerial vehicles (UAVs) have increasingly been used in various application areas, such as in the remote sensing or surveying. For these applications the UAV has to be equipped with a mapping sensor, which is mostly a camera. Furthermore, a georeferencing of the UAV platform and/or the acquired mapping data is required. The most efficient way to realize this georeferencing is the direct georeferencing, which is based on an onboard multi-sensor system. In recent decades, direct georeferencing systems have been researched and used extensively in airborne, ship and land vehicle applications. However, these systems cannot easily be adapted to UAV platforms, which is mainly due to weight and size limitations.

In this paper a direct georeferencing system for micro- and mini-sized UAVs is presented, which consists of a dual-frequency geodetic grade OEM GPS board, a low-cost single-frequency GPS chip, a tactical grade IMU and a magnetometer. To allow for $\mathrm{cm}-\mathrm{level}$ position and sub-degree attitude accuracies, RTK GPS (real-time kinematic) and GPS attitude (GPS compass) determination algorithms are running on this system, as well as a GPS/IMU integration.

Beside the direct georeferencing, also the precise time synchronization of the camera, which acts as the main sensor for mobile mapping applications, and the calibration of the lever arm between the camera reference point and the direct georeferencing reference point are explained in this paper. Especially the high accurate time synchronization of the camera is very important, to still allow for high surveying accuracies, when the images are taken during the motion of the UAV.

Results of flight tests demonstrate that the developed system, the camera synchronization and the lever arm calibration make directly georeferenced UAV based single point measurements possible, which have cm-level accuracies on the ground.
\end{abstract}

\section{INTRODUCTION}

For some years now, an increasing use of unmanned aerial vehicles (UAVs) is visible in many different application areas. Examples can be found in the areas of precision farming, infrastructure inspection, remote sensing or surveying. The reasons for the growing popularity of UAVs are that they simplify and improve many existing operations and that they enable new perspectives, which have sometimes even been unattainable so far.

Since there exist UAVs in very different weight and size classes, we like to point out that this paper is focused on micro- and minisized aerial vehicles, having a weight limit of $5 \mathrm{~kg}$ and a size limit of $1.5 \mathrm{~m}$ (Eisenbeiss, 2009). Especially the weight limit is important for us, since this weight limit corresponds to the requirements, which are written in the German aviation security act.

\subsection{Georeferencing of UAVs}

For many of the UAV applications, especially in the area of remote sensing and surveying, a high-accurate georeferencing of the UAV platform and/or the collected mapping data (e.g. images or laserscan point clouds), is usually required. This georeferencing includes the $\mathrm{cm}$-accurate determination of the $3 \mathrm{D}$ position (e.g. $X, Y, Z$ ) and the sub-degree accurate $3 \mathrm{D}$ attitude (e.g. $\alpha, \beta, \gamma)$ of the UAV relative to the earth, represented in a predefined coordinate frame. Therein, the attitude usually describes the rotation between the body fixed coordinate frame and the navigation coordinate frame.

One option to realize the georeferencing of UAV mapping data is the indirect georeferencing, based on ground control points (GCPs). However, depending on the object of interest the installation and measurement of GCPs is very time consuming and sometimes also impossible, e.g. when the object of interest is inaccessible. Furthermore, the detection and the allocation of the
GCPs can only be partially automated and is not real-time capable so far. Finally, an indirect georeferencing also requires a high overlapping image acquisition and a non-uniform surface texture. Summarizing, an indirect georeferencing is often ineffective and sometimes even not applicable.

The second option to realize the georeferencing of UAV mapping data is the direct georeferencing, which is based on an onboard multi-sensor system. In recent years, direct georeferencing systems have been extensively researched and used, in airborne, ship and land vehicle applications (Schwarz et al., 1993, Skaloud, 1999, Heipke et al., 2002). However, these systems cannot easily be adapted to small and lightweight UAVs, which is mainly due to the weight and size constraints. This is why the interest in small and lightweight direct georeferencing systems is growing simultaneously with the increasing interest in UAVs.

\subsection{Related work}

Usually, commercial-of-the-shelf micro- and mini-sized UAVs are already equipped with direct georeferencing sensors, such as a single-frequency GPS (Global Positioning System) chip, providing GPS C/A code-based positions, a low-cost IMU, a barometer and a magnetometer (Yoo and Ahn, 2003). However, this sensor combination only leads to position accuracies of approx. 5-10 m and attitude accuracies of approx. 1-5 deg, which in fact is good enough to realize an automated waypoint flight, but is insufficient for most of the remote sensing or surveying applications.

First approaches leading to a cm-accurate positioning of UAVs can be found in (Bláha et al., 2011, Rieke et al., 2011, Bäumker et al., 2013, Rehak et al., 2014). All of these approaches are scientific and non-industrial products. The cm-level position accuracies in these products are mostly based on RTK GPS (real-time kinematic GPS). Nevertheless, none of these products provides the position in real-time onboard of the UAV.

First commercial direct georeferencing systems, which are UAV 
capable, have only recently come onto the market. Examples are the Ellipse-D system from SBG (SBG, 2015) or the xNAV system from Oxford Technology Solutions (OXTS, 2015).

\subsection{Objectives}

In the recent years, we developed a direct georeferencing system for micro- and mini-sized UAVs, which has the following characteristics:

- Small and lightweight: The weight of the system is less than $500 \mathrm{~g}$, to be applicable on UAVs.

- High accuracies: The system is intended to provide $\mathrm{cm}$-level accurate positions and sub-degree accurate attitudes with a rate of $100 \mathrm{~Hz}$ during flights.

- Real-time capability: The position and attitude determination is performed in real-time onboard of the UAV. The mapping sensor (camera) can be clock synchronized with a sufficient accuracy.

- High reliability: Outages of single sensors can be bridged by other sensors.

- High flexibility: The system allows for the integration of additional external sensors (e.g. stereo cameras, laser scanner).

- Fast ambiguity resolution: Especially in kinematic applications, the GPS carrier phase ambiguities have to be resolved as fast as possible to allow for $\mathrm{cm}$-accurate baseline determinations.

One advantage that we still have with this system is the high flexibility in the hardware and the algorithm development, since an own developed system can easier be adapted to different and special applications.

The objective of this paper is the test and the evaluation of this system for the use of a direct georeferencing of aerial images, taken during flights with a micro-sized UAV. In the following section (section 2), we first describe the system design, which includes the UAV, the direct georeferencing system, the camera and the camera synchronization. Afterwards, in section 3, results of flight tests will be presented. Thereby, the focus lies on the evaluation of the ground accuracies and the accuracies of the directly georeferenced camera positions.

\section{SYSTEM DESIGN}

Some details to the developed direct georeferencing system have already been presented in (Eling et al., 2014b) and in (Eling et al., 2014c). However, in these publications, the UAV has not been equipped with a consumer camera. On this account, the new UAV setup and the precise synchronization of the consumer camera will be described in detail in the following subsections. The main characteristics of the direct georeferencing system will be summarized.

\subsection{UAV platform}

Meanwhile, there are various UAV platforms available on the market. Nevertheless, most of these UAVs are not suitable to be extended with additional sensors or to realize any modifications of their basic framework. We decided to use a Mikrokopter Okto XL construction kit (Hi Systems) for our developments. The advantages of this system are that the design is easy to modify, software and hardware customizations are possible and it is not too

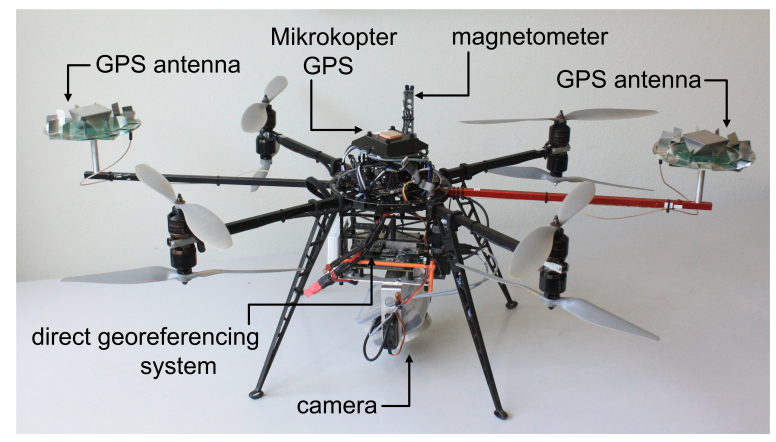

Figure 1: The UAV, equipped with direct georeferencing and mapping sensors.

expensive

The developed UAV platform is shown in Fig. 1. In contrast to the UAV, which has been presented in (Eling et al., 2014b) and in (Eling et al., 2014c), it is constructed with a coaxial setup. With this setup the system is more compact and there remain more possibilities, where sensors can be mounted.

To ensure more stability during flights, the centerplates, which hold the frame together, are made from carbon. On the top of the middle of the frame, a low-cost single-frequency GPS antenna is mounted, which is a standard Mikrokopter GPS. This antenna provides m-level accurate C/A code GPS positions, which are used for the navigation of the UAV during automated waypointflights. The GPS antennas, which are used for the more accurate direct georeferencing with our system, are mounted at the front and at the back of the body-frame x-axis of the UAV. The rear antenna provides the GPS raw observations for the RTK GPS positioning. Together with the front antenna both antennas form a short onboard GPS baseline on the UAV, which is used for the attitude determination (GPS compass).

The direct georeferencing system is placed below the centerplates and the consumer camera below the direct georeferencing system. The lever arm between the position, which is determined with the direct georeferencing system, and the camera reference point has to be calibrated and regarded (section 3.3).

The dimensions of the UAV are $1.0 \mathrm{~m} \times 0.8 \mathrm{~m} \times 0.35 \mathrm{~m}$, where the longest dimension points into the direction of the $\mathrm{x}$-axis of the body-frame. The total weight, including the Lithium Polymer batteries for the power supply, is $3.6 \mathrm{~kg}$, which complies with the German law formalities. The average flight time is $10-15 \mathrm{~min}$, depending on the wind, the application and the capacity of the batteries.

\subsection{Direct georeferencing system}

The dimensions of the direct georeferencing system are $11.0 \mathrm{~cm}$ $\times 10.2 \mathrm{~cm} \times 4.5 \mathrm{~cm}$ and the weight is $240 \mathrm{~g}$ without the GPS antennas (Fig.2). In Fig. 3 a flow chart of the system, including the sensors and the main calculation steps, is shown.

As the main positioning device, the system contains a geodetic grade dual-frequency OEM GPS board (Novatel OEM 615). Together with the GPS carrier phase measurements $\phi$ and the pseudorange measurements $P$ of a master station, which remains on the ground, RTK GPS positions of the UAV can be determined in real-time onboard of the UAV. The transfer of the data from the base station to the direct georeferencing system is realized by radio modules (XBEE Pro 868).

The data of the dual-frequency GPS board is also used for the attitude determination. For this attitude determination, also a lowcost single-frequency GPS chip (Ublox LEA6T) is integrated in the system. The GPS antennas, which correspond to the both GPS receivers, form a short baseline on the UAV $(92 \mathrm{~cm})$ (Fig 1), which acts as a single-frequency GPS compass. 


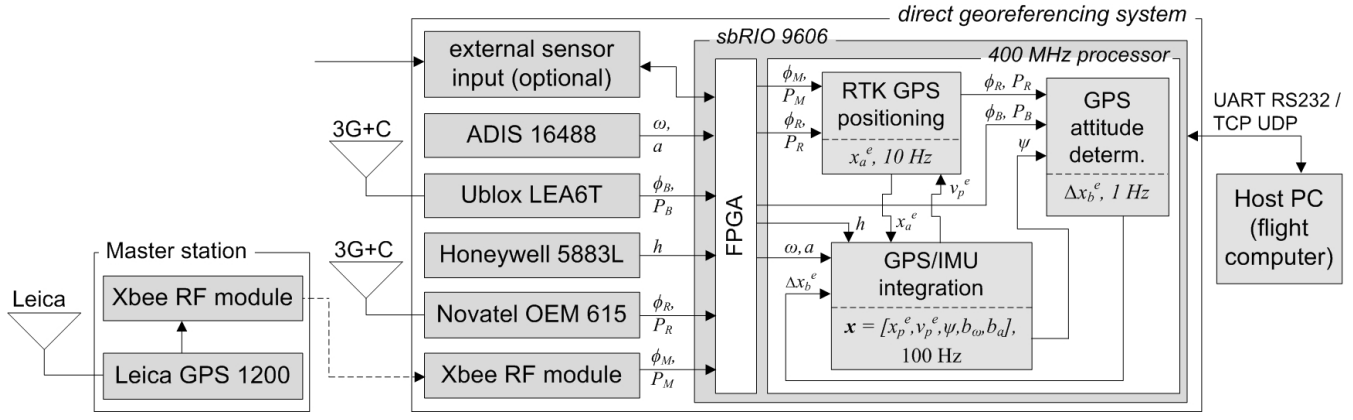

Figure 3: Flow-chart of the direct georeferencing system, where $\phi$ stands for GPS carrier phases and $P$ for GPS pseudoranges. $\omega$ denotes angular rates, $a$ accelerations and $h$ magnetic field observations. $\psi$ is the estimated attitude.

Even if only one dual-frequency GNSS antenna is required, both GPS antennas are geodetic grade dual-frequency GNSS antennas (NavXperience $3 \mathrm{G}+\mathrm{C}$ ), which provide high-quality GPS observations. Since these antennas are originally too heavy to be used on a UAV, we decided to dismantle these antennas, with the result that all unnecessary weight (e.g. the housing and the 5/8" thread) could be omitted. Afterwards the antennas were re-calibrated in our anechoic chamber (Zeimetz and Kuhlmann, 2010).

A tactical grade MEMS IMU (Analog devices ADIS16488), containing 3-axis gyroscopes, accelerometers, magnetometers and a barometer, provides several observations, which can be used for the position and attitude determination. Particularly, we use the angular rates $\omega$ and the accelerations $a$ from the IMU. Since the internal magnetometers of the IMU are too much affected by the electric currents of the UAV, an additional magnetometer (Honeywell HMC5883L) is mounted on the end of one of the rotor-free UAV arms (Fig. 1).

Beside the sensors, the direct georeferencing system also consists of a processing unit (National Instruments, sbRIO 9606), which is a reconfigurable IO board, including an FPGA (field programmable gate array) and a $400 \mathrm{MHz}$ processor. In this combination, the FPGA is used for a fast and parallel communication with the sensors. Afterwards, the preprocessed sensor data are provided to the $400 \mathrm{MHz}$ processor via direct memory accesses, avoiding delays and supporting the real-time capabilities of the system. Finally, the actual position and attitude determination is carried out on the $400 \mathrm{MHz}$ processor.

\subsection{Position and attitude determination algorithms}

The position and attitude determination algorithms can be subdivided into (i) the RTK GPS positioning, (ii) the GPS attitude baseline determination and (iii) the GPS/IMU integration (Fig.3). All the algorithms, which are running on the real-time processing unit, are in-house developed. Generally, the GPS/IMU integration could be realized in one tightly coupled approach. Nevertheless, in the current implementation, we decided to separate the

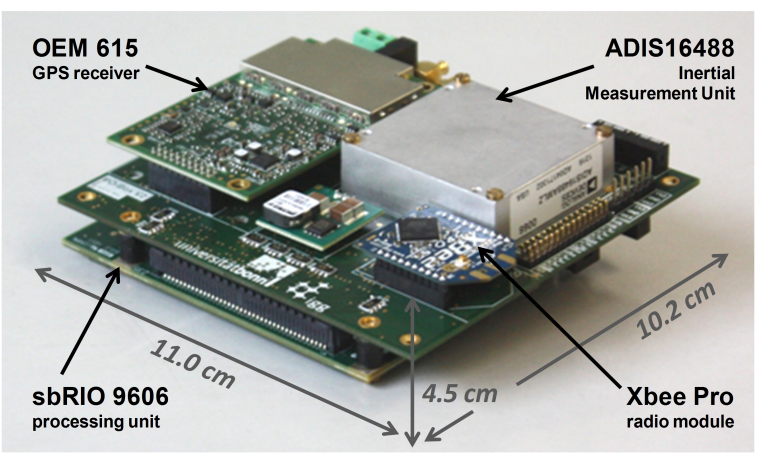

Figure 2: The direct georeferencing system, with its components. different raw data calculation steps and we only use interactions at the level of parameters. This approach has the advantage that the integration is more reliable and more practical in the real-time programming.

2.3.1 GPS/IMU integration: In the GPS/IMU integration all available sensory input is fused for the position and attitude determination. Therein the GPS and the IMU measurements complement each other well, since the IMU provides short-term stable high-rate $(100 \mathrm{~Hz})$ data and the GPS provides long-term-stable low-rate $(1-10 \mathrm{~Hz})$ data.

In principal, the GPS/IMU integration consists of the Strapdown Algorithm, where the high dynamic movement of the system is determined integrating the angular rates and the accelerations of the MEMS IMU in real-time, and the Kalman filter update, which bounds the drift of the inertial sensor integration. The Kalman filter update is realized in an error state space Kalman filter.

In the system model of the Kalman filter, the navigation equations of the body-frame (b-frame) are expressed in an earth-fixed frame (e-frame). The full state vector $\mathbf{x}$ includes the position $\mathbf{x}_{p}^{e}$ and the velocity $\mathbf{v}_{p}^{e}$, represented in the e-frame. For the attitude determination a quaternion $\mathbf{q}$ is used. Finally, the accelerometer bias $\mathbf{b}_{a}^{b}$ and the gyro bias $\mathbf{b}_{\omega}^{b}$ are also estimated:

$$
\mathbf{x}=\left[\begin{array}{lllll}
\mathbf{x}_{p}^{e T} & \mathbf{v}_{p}^{e T} & \mathbf{q}^{T} & \mathbf{b}_{a}^{b^{T}} & \mathbf{b}_{\omega}^{b^{T}}
\end{array}\right] .
$$

The observations in the measurement model are:

(a) the RTK GPS position $\mathbf{x}_{a}^{e}$ of the dual-frequency RTK GPS antenna reference point, expressed in the e-frame, (section 2.3.2), (b) the GPS attitude baseline vector $\Delta \mathbf{x}_{b}^{e}$, expressed in the eframe (section 2.3.3) and

(c) the magnetic field vector $\mathbf{h}^{b}$, measured in the b-frame.

Ferromagnetic material on the UAV and the high electric currents of the rotors lead to significant disturbances of the magnetometer during flights. While the influence of the ferromagnetic material can be compensated by calibration procedures (Klingbeil et al., 2014), the influence of the dynamically changing electric currents are more challenging. This is the reason why the GPS compass is also realized on the UAV, which improves the yaw angle determination significantly.

2.3.2 RTK GPS positioning: The RTK GPS positions, which are inputs to the GPS/IMU integration algorithms, are calculated in real-time with a rate of $10 \mathrm{~Hz}$. Details to the implemented RTK-GPS algorithms can be found in (Eling et al., 2014a) and (Eling et al., 2014b).

Summarizing, the parameter estimation of the RTK GPS positioning is realized in a full state extended Kalman filter. Beside the rover position $\mathbf{x}_{a}^{e}=\left[\begin{array}{lll}x_{a, x}^{e} & x_{a, y}^{e} & x_{a, z}^{e}\end{array}\right]$, represented in the $\mathrm{e}$-frame, the EKF state vector $\mathbf{x}_{S D}$ also contains single-difference (SD) ambiguities $N^{j}$ on the GPS L1 and the GPS L2 frequency.

$$
\mathbf{x}_{S D}=\left[\begin{array}{lll}
\mathbf{x}_{a}^{e} & N_{L 1}^{j} & N_{L 2}^{j}
\end{array}\right]^{T} .
$$


To allow for an instantaneous ambiguity resolution the observation vector $\mathbf{y}$ consists of double-difference (DD) carrier phases $\phi_{R M}^{j k}$ and DD pseudoranges $P_{R M}^{j k}$ on the GPS L1 and the GPS L2 frequency:

$$
\mathbf{y}=\left[\begin{array}{llll}
\phi_{R M, L 1}^{j k} & \phi_{R M, L 2}^{j k} & \mathbf{P}_{R M, L 1}^{j k} & \mathbf{P}_{R M, L 2}^{j k}
\end{array}\right]^{T} .
$$

As system dynamics model a random walk model is used, which is a simple model, but agrees with the movement of every vehicle, when the process noise is chosen appropriate. After the ambiguities are estimated as real values, they have to be fixed to integers in the ambiguity resolution. For this, the MLAMBDA method is applied (Chang et al., 2005).

Usually, the ambiguity resolution with the above described algorithm takes a few epochs, but often the ambiguities can already be fixed instantaneously. Due to the GPS/IMU integration, cycle slips can be detected and repaired very reliably (Eling et al., 2014b).

2.3.3 GPS attitude determination (GPS compass): A significant challenge in the processing of the attitude baseline is the ambiguity resolution, since only single-frequency GPS observations can be used. Empirical tests have shown that the ambiguity resolution of a single-frequency GPS baseline generally takes several minutes (Odijk et al. 2007). To improve the ambiguity resolution and to actually enable an instantaneous ambiguity fixing during kinematic applications, we use, among other strategies, the additional information from the magnetometer and the GPS/IMU integration.

As well as in the RTK GPS positioning, the SD ambiguities $N^{j}$ of the GPS attitude baseline are estimated via a further full state extended Kalman filter. The state vector consists of the baseline parameters $\Delta \mathbf{x}_{b}^{e}$ and the first deviation of the baseline parameters $\Delta \dot{\mathbf{x}}_{b}^{e}$ :

$$
\mathbf{x}_{S D}=\left[\begin{array}{lll}
\Delta \mathbf{x}_{b}^{e} & \Delta \dot{\mathbf{x}}_{b}^{e} & N_{L 1}^{j}
\end{array}\right]^{T} .
$$

As observations DD carrier phases $\phi_{R M}^{j k}$ and DD pseudoranges $P_{R M}^{j k}$ on the GPS L1 frequency are used. Furthermore, to improve the ambiguity resolution the attitude from the GPS/IMU integration is added to the observation vector, by transforming the known b-frame baseline parameters into the e-frame: $\Delta \overline{\mathbf{x}}_{b}^{e}$. Finally, also the known baseline length $s$ can be added as a constraint to the observation vector $\mathbf{y}$ :

$$
\mathbf{y}=\left[\begin{array}{llllll}
\phi_{R M, L 1}^{j k} & \mathbf{P}_{R M, L 1}^{j k} & \Delta \bar{x}_{b, x}^{e} & \Delta \bar{x}_{b, y}^{e} & \Delta \bar{x}_{b, z}^{e} & s
\end{array}\right]^{T} .
$$

Due to the prior information $\Delta \overline{\mathbf{x}}_{b}^{e}$ about the attitude of the baseline, the real valued ambiguities can already be estimated with high accuracies in the Kalman filter. Therefore, in more than $80 \%$ of the epochs, the ambiguities can already be fixed to integer values in the first epoch, applying the MLAMBDA method. If the ambiguities could not be fixed, further investigations are necessary. Therefor, we use the ambiguity function method (Eling et al., 2013) and the known baseline length, to exclude false candidates from the ambiguity search space. Tests have shown, that our approach leads to an instantaneous ambiguity resolution success rate of $95 \%$ during UAV flights.

With the ambiguities fixed, the baseline parameters can be determined with $\mathrm{mm}$ - to $\mathrm{cm}$-accuracies. With the baseline length of $92 \mathrm{~cm}$, this leads to yaw angle accuracies in the range of $0.2-$ $0.5 \mathrm{deg}$.

\subsection{Camera and camera synchronization}

The used onboard camera is a Panasonic Lumix GX1 consumer system camera. The size of this camera is $11.6 \times 6.8 \times 3.9 \mathrm{~cm}$

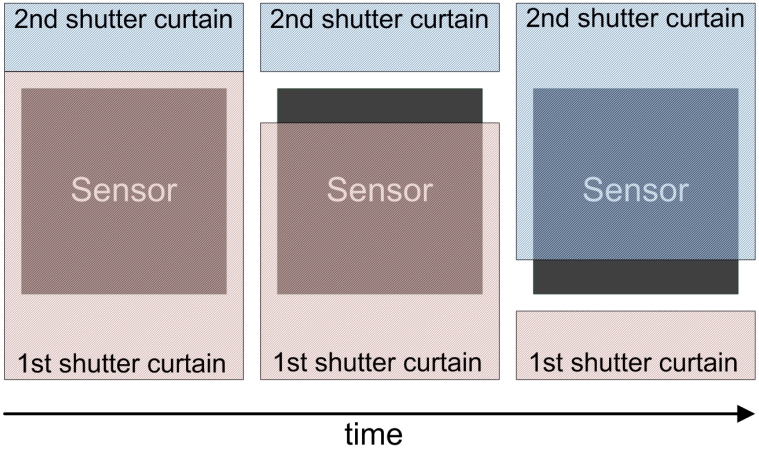

Figure 4: The functionality of a mechanical focal-plane shutter.

and the weight of the body is $320 \mathrm{~g}$. Therefore, it is well suited for UAV applications. The camera includes a mechanical focalplane shutter, has a 15.8 megapixel sensor and is equipped with a $20 \mathrm{~mm}$ fixed focal length.

For the direct georeferencing of moving systems, the time synchronization between the mapping sensor and the trajectory estimation is crucial. Small deviations in the time synchronization can lead to large deviations in the georeferencing. For example, if a UAV flies with a speed of $3 \mathrm{~m} / \mathrm{s}$ and the synchronization error is $10 \mathrm{~ms}$, the positioning error would be $3 \mathrm{~cm}$.

In principal, there exist two possibilities to realize the time synchronization between a consumer camera and a direct georeferencing system:

- Trigger time: The camera is triggered by the direct georeferencing system and the respective trigger time is used for the time synchronization.

- Exposure start time: The camera is triggered by any mechanism and the exposure start time of the camera is used for the time synchronization.

From a technical point, the triggering of a consumer camera is not very difficult, if the camera has an external camera trigger. However, the drawback of using the trigger time for the time synchronization is that the trigger time does usually not agree with the exposure start time. Although there are possibilities to reduce the time delay between the camera triggering and the image exposure, this effect can not be eliminated. Thus, when the trigger time should be used for the time synchronization, a calibration has to be performed, to determine the time delay between the triggering and the recording of an image (Rehak et al., 2014).

To determine the time delay of our camera, we developed a LED clock (light-emitting diode), which consists of a $8 \times 8$ LED matrix (see Fig.4). Every single LED of this matrix can be turned on with a microsecond time resolution in real-time. For a delay measurement, the LED clock is started simultaneously with the camera trigger. By means of the LED, which is turned on in the recorded image, the time delay between the triggering and the recording of the image can be determined.

Due to many of the LED-clock measurements, we could determine the time delay of our camera. However, the tests have also shown that the time delay between the triggering and the recording is not constant, even for fixed camera settings. The variations of the time delay are in the range of $\pm 10 \mathrm{~ms}$ under good conditions, which is too much, when the images should be taken during the motion of the UAV.

When tapping the exposure start time signal directly, one would avoid the unknown and varying delay between the trigger and the 


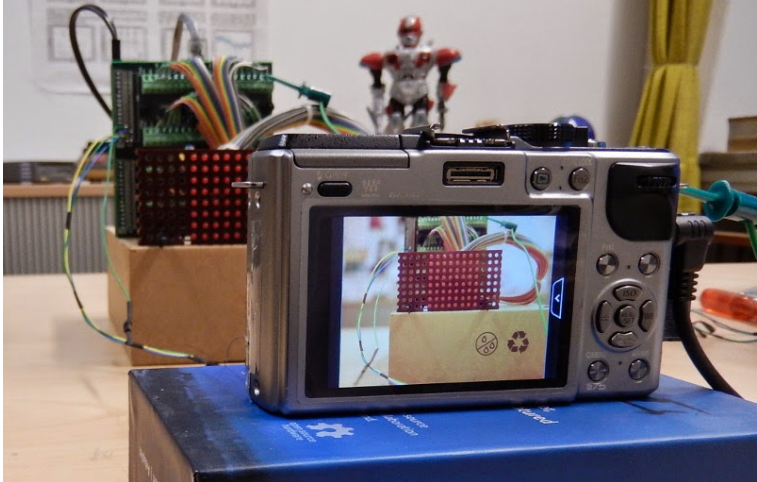

Figure 5: Calibration of the time delay between the shutter signal and the actual exposure.

exposure. However, in this case, some modifications to the camera are necessary. As mentioned earlier, the GX1 camera exposures with a mechanical focal-plane shutter. The functionality of a focal-plane shutter is shown in Fig.4. The exposure starts, when the first shutter curtain moves downwards. Since the exposure has to be short during flights, the second shutter curtain begins to move downwards at a set distance from the first one, before the first shutter curtain arrives at the final position. Finally, the first shutter curtain finishes moving and the second shutter curtain is going to cover the sensor completely.

Aided by the data sheet and the electrical circuit diagram, it was possible to tap the signals of both shutter curtains on the printed circuit board of the camera. With the signal of the first shutter curtain the start of an image exposure can be assigned clearly. Therefore, this signal has been lead outside the camera with a cable, which can be connected to the direct georeferencing system, where the time is logged in hard real-time, when the synchronization signal arrives. Due to the mechanical realization of the shutter, there still remains a time delay between the shutter signal and the actual image exposure. This time delay had to be measured, to be able to correct it sufficiently. Therefor, the LED-clock was used again. The results have shown that the time delay is $3 \mathrm{~ms}$. Nevertheless, the decisive issue is that there was no variance of this time delay detectable.

The advantages of the shutter curtain signal synchronization are that the synchronization is independent on the camera settings. All the automatic modes of the camera, such as the autofocus or the automated exposure control, can be used, since trigger delays, caused by these functions, happen before the exposure. Also this method provides an information, if the image has actually been taken. A trigger signal is only the intention to take an image, which may still fail, e.g. if the autofocus was not successful. This would lead to incorrect assignments of the position/attitudes and images.

\section{FLIGHT TESTS}

Different flight tests were performed to evaluate the accuracy of the direct georeferencing. In this evaluation especially the ground accuracy is significant, since UAVs are mostly used to measure points or surfaces on the ground. Nevertheless, also the accuracy of the directly georeferenced camera positions are of interest. However, to be able to evaluate the direct georeferencing accuracies, first the lever arm between the camera reference point and the GPS/IMU reference point has to be determined.

During the test flights, the flight altitude was mostly in the range of $20 \mathrm{~m}$ and the average flight speed was $2 \mathrm{~m} / \mathrm{s}$. The images were always taken during the motion of the UAV.

\subsection{Test fields}

Two test fields with sizes of approx. $50 \times 50 \mathrm{~m}$ were established, to evaluate the performance of the direct georeferencing and to realize the calibration of the lever arm. Since the test fields are intended to be used for a direct and an indirect georeferencing, black and white targets were installed, which can be used as GCPs for an indirect georeferencing and as checkpoints for a direct georeferencing. Test field 1 contains 22 and test field 2 contains 9 black and white targets.

The coordinates of the targets were precisely measured with a tachymeter and transformed into the GPS-frame. This transformation was based on five points, where long-term dual-frequency GPS observations ( $4 \mathrm{~h}$ ) were acquired. The baselines between the GPS master station and the other four GPS stations were determined in a postprocessing phase. By combining, the GPS and the tachymeter measurements, the coordinates of the black and white targets could be determined with mm-accuracy. To ensure a high consistency between the coordinates of the targets and the direct georeferencing, the same GPS station was used as the master station for the direct georeferencing unit and for the coordinate determination of the black and white targets.

Since the quality of the GPS observations is important, not only for the measurement of the target coordinates, but also for the direct georeferencing during the flights, the test fields are located on an agricultural field with nearly unlimited visibility to the sky. Therefore, the GPS measurement conditions were always good during the test flights. In Fig. 6 an orthophoto of test field 1 is presented. Therein also the positions of the GCPs, the GPS stations and the tachymeter are marked. Beside the accuracy evaluations, presented in this paper, this field is also used to determine plant growth rates based on UAV data.

\subsection{Image processing}

During the UAV operations, the acquired images are stored on the SD card of the camera. The positions and attitudes of the UAV, which are determined in real-time and onboard of the UAV (section 2.3), are logged on a USB device, which is connected to the direct georeferencing system. This log data also includes the time

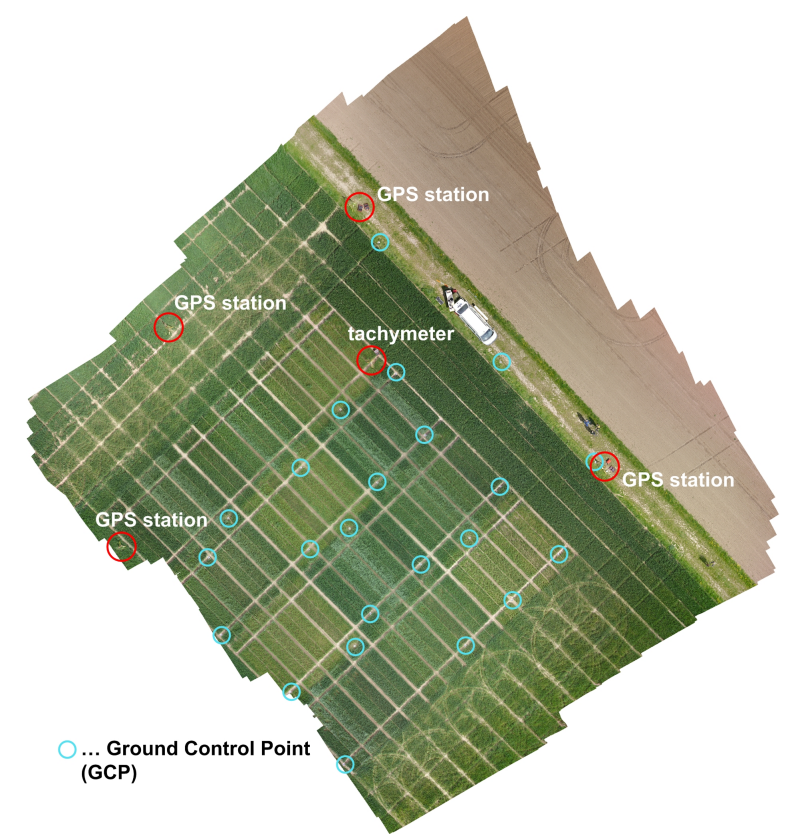

Figure 6: Orthophoto of one of the test fields. The red circles mark four of the five GPS stations and the tachymeter. The cyan circles mark the Ground Control points (GCPs). 


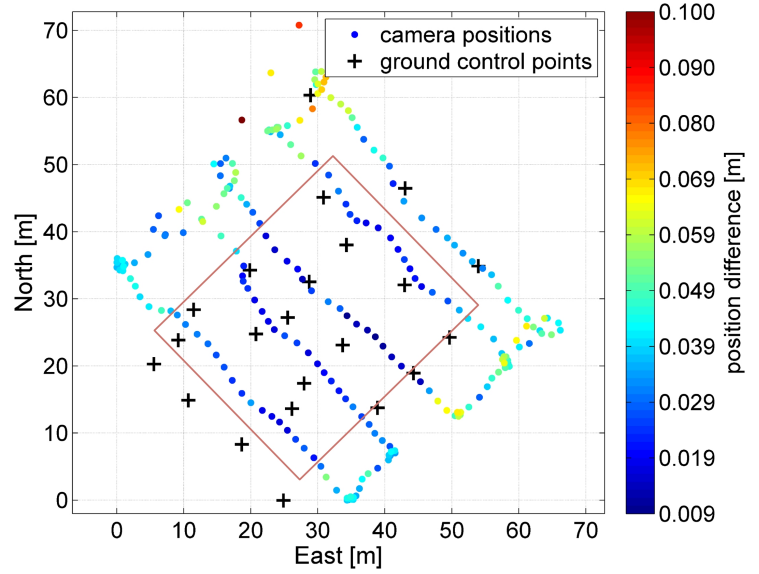

Figure 7: Difference between the directly and the indirectly georeferenced camera positions.

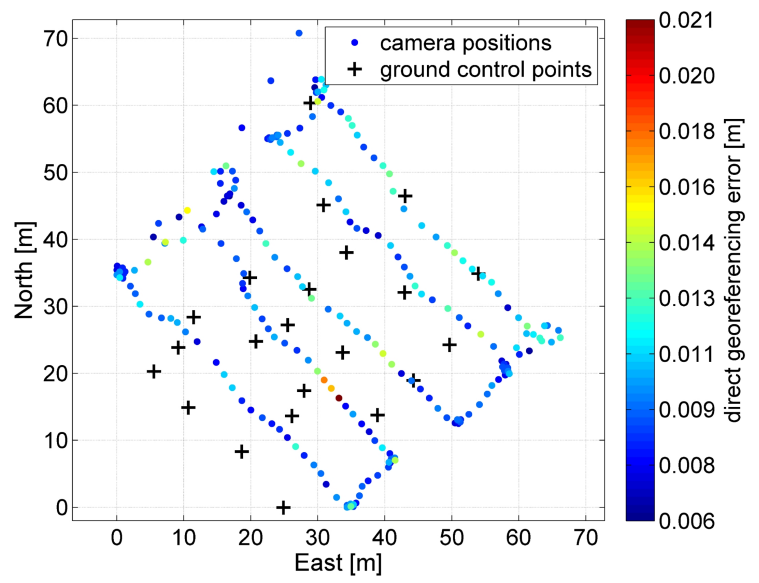

Figure 8: Error of the directly georeferenced camera positions.

deterministic camera synchronization signal (section 2.4). Therefore, a distinct assignment between the images and the positions and attitudes is possible. The actual image processing is realized in a postprocessing phase.

The software used here is the Pix4Dmapper of the company Pix 4 D. This software is a complete mapping and modeling solution, supporting geotags and GCPs for the georeferencing. For the direct georeferencing, we handed over the positions of the camera at the time of the image recording. Certainly, it is also possible to import the attitudes in Pix 4 D, but we wanted to prove the positiononly solution here, which is more practical for most users. As accuracies $2 \mathrm{~cm}$ for the East and North component and $3 \mathrm{~cm}$ for the Up (Height) component were set in the software. The reason, the height-error is set to a bigger value is that the directly georeferenced positions are mainly based on the RTK GPS positions.

\subsection{Lever arm calibration and camera position accuracy}

To have an unobstructed view, the camera has to be mounted below the UAV (see Fig. 1). On this account, a non-negligible lever arm between the camera reference point and the GPS/IMU reference point exists. This lever arm has to be known.

The determination of the lever arm has been realized with UAV flights, where images, positions and attitudes were acquired. Using this data, the lever arm could be determined, comparing the directly and the indirectly georeferenced UAV positions. While the indirectly georeferenced positions are the positions of the camera at the time of an image exposure, the directly georeferenced positions refer to the GPS/IMU reference point at the time
Table 1: Lever arm determination

\begin{tabular}{lccc}
\hline & East [m] & North [m] & Up [m] \\
\hline RMS & 0.010 & 0.014 & 0.011 \\
Max dev. & 0.023 & 0.033 & 0.029 \\
Min dev. & -0.018 & -0.027 & -0.022 \\
\hline
\end{tabular}

of an image exposure. Both positions are located in the same coordinate frame. The mean differences, between the indirectly georeferenced camera positions and the directly georeferenced positions, transformed into the body-frame, lead to the 3D lever

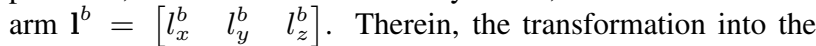
body-frame is based on the GPS/IMU attitude, which was determined by the direct georeferencing system.

In table 1, the RMS and the maximum and minimum deviations of the lever arm determination are presented. Accordingly, the RMS is approx. $1 \mathrm{~cm}$ and the deviations to the mean lever arm are in the level of $\pm 3 \mathrm{~cm}$. This is sufficient for this first test, but it also shows that there are improvements possible. These improvements would include a higher overlap of the images, the use of more GCPs and the acquisition of more images with different perspectives.

In Fig. 7, the differences between the directly and the indirectly georeferenced camera positions, at the respective recording positions, are shown. Therein, the calibrated lever arm has already been added to the direct georeferencing positions, using the attitude angles, which have been determined by the direct georeferencing system. The accuracies of these attitude angles are usually $<0.5 \mathrm{deg}$.

Fig. 7 clearly demonstrates that the deviations between the direct and the indirect georeferencing increase systematically outside the area, where the GCPs could be detected in many images with different perspectives (red rectangle). By comparison, the positioning error, which results from the estimated covariance matrix of the direct georeferencing, has been nearly constant and very small during this flight (Fig.8). The variations are in the range of 6-21 mm. This indicates that the systematic deviations, seen from Fig. 7, come from the indirect georeferencing. Therefore, only the central areas of the test flights were used for the lever arm determination.

\subsection{Ground accuracies}

With the direct georeferencing system, the camera synchronization and the known lever arm calibration, the positions of the camera can be determined directly at the recording time during UAV flights. This definitely improves the operating procedure of remote sensing and surveying applications with micro- and mini-sized UAVs and is an important step towards the automation of these processes. Nevertheless, still the question has to be answered, how accurate points on the ground can be measured, using the direct georeferencing system.

To answer this question the GCPs of both test fields were used as checkpoints for always one flight at each field. The differences between the a priori known mm-accurate coordinates of the checkpoints and the measured coordinates are shown in Fig. 9.

Accordingly, the deviations are mostly in the level of $\pm 5 \mathrm{~cm}$ for field 1 and $\pm 1.5 \mathrm{~cm}$ for field 2 . The RMS values of both flights are shown in table 2. Thus, the RMS is between 2.6 and $3.8 \mathrm{~cm}$ for flight 1 and between 0.6 and $0.9 \mathrm{~cm}$ for flight 2 . This

Table 2: Check point deviations for two test flights.

\begin{tabular}{lcccc}
\hline & \# points & East $[\mathrm{m}]$ & North $[\mathrm{m}]$ & $\mathrm{Up}[\mathrm{m}]$ \\
\hline RMS Field 1 & 17 & 0.038 & 0.026 & 0.030 \\
RMS Field 2 & 9 & 0.009 & 0.006 & 0.009 \\
\hline
\end{tabular}




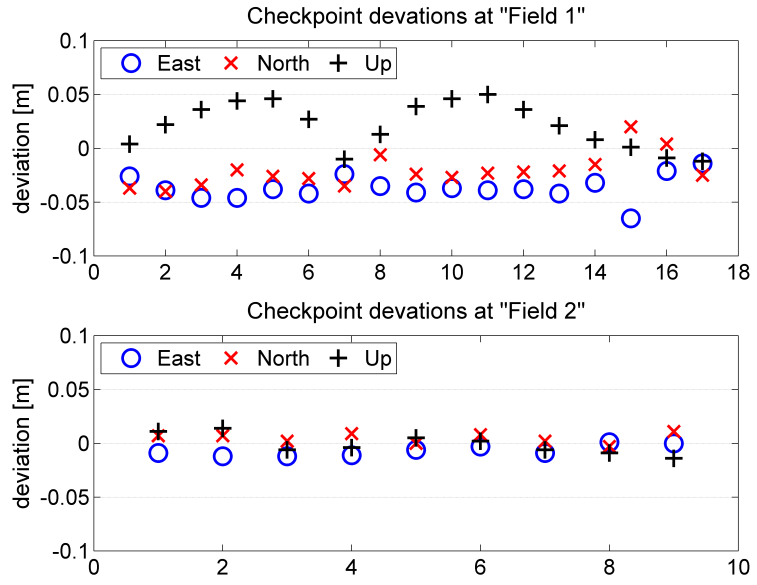

Figure 9: The deviations at the checkpoints of both test fields.

demonstrates that the direct georeferencing of UAV based mapping data can lead to $\mathrm{cm}$-accurate ground measurements during remote sensing and surveying applications, when the lever-arm between the camera and the direct georeferencing unit is calibrated and the camera is synchronized with a sufficient accuracy.

\section{CONCLUSION}

In this paper a direct georeferencing system was presented, which is mainly intended to be used for the real-time position and attitude determination of micro- and mini-sized UAVs. Summarizing, the system has the following characteristics: (i) The weight of the system is $240 \mathrm{~g}$, without the GPS antennas. (ii) The system is real-time capable. (iii) All sensors are synchronized and/or hardware triggered. (iv) The system provides high accuracies for the positions $\left(\sigma_{\text {pos }}<5 \mathrm{~cm}\right)$ and attitudes $\left(\sigma_{a t t}<1 \mathrm{deg}\right)$ during flights. (v) The integration of additional sensors, such as cameras, is possible.

Due to a modification to the used consumer camera, which acts as the mapping sensor in our setup, a highly accurate $(<1 / 10 \mathrm{~ms})$ camera synchronization could be realized, which uses the first shutter curtain signal of the mechanical shutter of the camera, to determine the correct time of the image exposure.

Test flights, where several directly georeferenced point measurements on the ground were compared to known coordinates, have shown that the direct georeferencing system leads to point measurement accuracies of less than $5 \mathrm{~cm}$ on the ground. That shows us that the developed direct georeferencing system is well suited to improve the operating procedure of remote sensing and surveying applications with micro- and mini-sized UAVs.

\section{ACKNOWLEDGMENTS}

This work was funded by the DFG (Deutsche Forschungsgemeinschaft) under the project number 1505 "Mapping on Demand". The authors wish to express their gratitude for this support.

\section{REFERENCES}

Bäumker, M., Przybilla, H.-J. and Zurhorst, A., 2013. Enhancements in UAV flight control and sensor orientation. In: The International Archives of Photogrammetry, Remote Sensing and Spatial Information Science XL-1/W2, UAV-g 2013, pp. 33-38.

Bláha, M., Eisenbeiss, H., Grimm, D. and Limpach, P., 2011. Direct georeferencing of UAVs. In: The International Archives of Photogrammetry, Remote Sensing and Spatial Information Science XL-1/W2, UAV-g 2011, pp. 1-6.
Chang, X.-W., Yang, X. and Zhou, T., 2005. Mlambda: A modified lambda method for integer least squares estimation. Journal of geodesy 79, pp. 552-565.

Eisenbeiss, H., 2009. UAV Photogrammetry. Phd thesis, no. 18515, ETH Zurich.

Eling, C., Heinz, E., Klingbeil, L. and Kuhlmann, H., 2014a. Cycle slip detection in the context of RTK GPS positioning of lightweight UAVs. In: 4th International Conference on Machine Control \& Guidance, Braunschweig, Germany, pp. 148-155.

Eling, C., Klingbeil, L. and Kuhlmann, H., 2014b. Development of an RTK-GPS system for precise real-time positioning of lightweight UAVs. In: A. Wieser (ed.), Ingenieurvermessung 2014, Wichmann Verlag, pp. 111-123.

Eling, C., Klingbeil, L., Wieland, M. and Kuhlmann, H., 2014c. Direct georeferencing of micro aerial vehicles - system design, system calibration and first evaluation tests. PFG - Photogrammetrie, Fernerkundung, Geoinformation 4, pp. 227-237.

Eling, C., Zeimetz, P. and Kuhlmann, H., 2013. Development of an instantaneous gnss/mems attitude determination system. GPS Solution 17, pp. 129-138.

Heipke, C., Jacobsen, K. and Wegmann, H., 2002. Integrated sensor orientation - test report and workshop proceedings. In: OEEPE Official Publication 43, Frankfurt/Main.

Klingbeil, L., Eling, C., Zimmermann, F. and Kuhlmann, H., 2014. Magnetic field sensor calibration for attitude determination. Journal of Applied Geodesy 8(2), pp. 97-108.

OXTS, 2015. xnav, http://www.oxts.com/products/xnav/.

Rehak, M., Mabillard, R. and Skaloud, J., 2014. A micro aerial vehicle with precise position and attitude sensors. PFG - Photogrammetrie, Fernerkundung, Geoinformation 4, pp. 239-251.

Rieke, M., Foerster, T., Geipel, J. and Prinz, T., 2011. Highprecision positioning and real-time data processing of uavsystems. In: International Archives of the Photogrammetry, Remote Sensing and Spatial Information Sciences, Vol. XXXVIII (1-C22), UAV-g conference, Zurich, Switzerland.

SBG, 2015. Ellipse-d: Miniature dual gps ins, http://www.sbgsystems.com/products/ellipse-d-dual-antenna-gps-inertialsystem.

Schwarz, K.-P., Chapman, M., Cannon, M. and Gong, P., 1993. An integrated INS/GPS approach to the georeferencing of remotely sensed data. Photogrammetric Engineering \& Remote Sensing 59(11), pp. 1667-1674.

Skaloud, J., 1999. Optimizing Georeferencing of Airborne Survey Systems by INS/DGPS. PhD thesis, University of Calgary, Canada.

Yoo, C. and Ahn, I., 2003. Low cost gps/ins sensor fusion system for uav navigation. In: The 22nd Digital Avionics Systems Conference, Vol. 2, pp. 8.A.1-1 - 8.A.1-9.

Zeimetz, P. and Kuhlmann, H., 2010. Validation of the laboratory calibration of geodetic antennae based on gps measurements. In: FIG Working Week, 11.-16. April, Sydney, Australia. 